\title{
Text Retrieval Oriented Auto-construction of Conceptual Relationship
}

\author{
Yi Hu${ }^{1}$, Ruzhan $\mathrm{Lu}^{1}$, Yuquan Chen ${ }^{1}$, and Bingzhen Pei ${ }^{1,2}$ \\ ${ }^{1}$ Department of Computer Science and Engineering, \\ Shanghai Jiao Tong University, Shanghai, China \\ ${ }^{2}$ School of Computer Science and Engineering, \\ Guizhou University, Guiyang, China \\ \{huyi, rz-lu, yqchen, peibz\}@ics.sjtu.edu.cn
}

\begin{abstract}
The dependence analysis is usually the key for improving the performance of text retrieval. Compared with the statistical value of a conceptual relationship, the recognition of relation type between concepts is more meaningful. In this paper, we explored a bootstrapping method for automatically extracting semantic patterns from a large-scale corpus to identify the geographical "is part of" relationship between Chinese location concepts. The experiments showed that the pattern set generated by our method achieves higher coverage and precision than DIPRE does.
\end{abstract}

Keywords: Conceptual Relationship; Extraction Pattern; Text Retrieval.

\section{Introduction}

The independence assumption has been widely used in current retrieval models. Although the assumption makes the models easily to build up, the independence between words in language is obviously trustless in fact. Naturally, a promising idea based on accurate conceptual relationship for information retrieval has been tried in [4]. Apparently, the deep understanding of content is closer to human cognition. Note that in terms of information retrieval based on concepts, it needs recognizing all valid relation types between concepts to build up a complete conceptual graph of content, and then providing response to the users' requirement.

Seen from the previous efforts, unsupervised bootstrapping had been used in many fields of information extraction. With respect to recognizing concept types (weapon names, terrorism organizations etc.), the efforts in [3][5] are significant efforts. On the other hand, extracting conceptual pairs with certain relationships from English corpus are also tested [1][2]. This paper also proposes a method for constructing relationship under bootstrapping learning by taking the geographical "is part of" as example.

\section{Our Contributions}

Our method is implemented based on the idea of DIPRE [2] by proposing a new SPG (Semantic Pattern Getter) system. The contributions of our work lie in: 
1. Introducing the bi-sequence alignment algorithm in bio-informatics to extract multiple common subsequences (MCS) for getting flexible context expression rather than the single longest common subsequence (LCS) in DIPRE.

2. Defining a new evaluation metric for the pattern confidence, which improves the extracting quality.

\subsection{Generating Patterns}

A formalized pattern is a five-tuple, i.e. (prefix, middle, suffix, order, confidence) that has the same meaning of [2]. The system captures patterns from the contexts of given seeds, and the procedure of generating patterns in our study is illustrated as:

Step 1. Find all the occurrences of every seed in the large-scale corpus and record the left, middle and right strings of the context.

Step 2. Use the bi-sequence alignment algorithm to extract patterns. Each context pair of a seed generates a candidate pattern.

Step 3. The candidate patterns are filtered through the pattern validation rules.

Because there is the detailed description in [6], we do not describe the alignment algorithm in details. But we can see an instance generated from two contexts:

Context1: 公司地址: 中国上海市江浦路1515号。

(Company Address: No. 1515, Jiang Pu Rd., Shanghai City, China.)

Context2: 公司通信地址: 中国上海市国顺路549号。

(Company Corresponding Address: No.549, Guo Shun Rd., Shanghai City, China.) The bi-sequence alignment algorithm extracts a pattern denoting the "is part of" relationship between two location concepts: (公司 $<A N Y \_S T R I N G>$ 地址 : , NULL, 市 $<A N Y$ STRING $>$ 路 $<A N Y \_S T R I N G>$ 号。, -1 , <Confidence $>$ ). In order to make the pattern not to be overly generalized, we define the following validation rules to choose new candidate patterns with higher qualities.

Rule 1. The "prefix" or "suffix" cannot be just a $<$ ANY_STRING >;

Rule 2. Both the most right component of "prefix" and the most left component of "suffix" cannot be an "<ANY_STRING>";

Rule 3. The "prefix", "middle" and "suffix" cannot just be punctuation;

These rules are simple and easy to understand. They are the experiences from extracting patterns. Note that, the system removes those Chinese stop-words, such as “的(de)" and “了(le)". In terms of a context set containing $N$ sentences, the procedure can at most generate $C_{N}^{2}$ candidate patterns. But a lot of them will be removed after using the validation rules.

\subsection{Pattern Confidence}

How to evaluate the pattern confidence is the key factor affecting the coverage and precision of final pattern set. We define a new confidence definition in (1).

$$
\operatorname{Conf}_{\text {RlogF }}(P)=\left(\frac{P_{\text {positive }}}{P_{\text {positive }}+P_{\text {negative }}}\right) \times\left[\log _{2}\left(P_{\text {new }}+1\right)\right]^{\alpha}
$$


Where, $P$ denotes the evaluated pattern and $P_{\text {positive }}$ denotes the number of correct pairs in all the extracted pairs in current iteration; $P_{\text {negative }}$ denotes the number of wrong pairs; $P_{\text {new }}$ is the number of newly extracted pairs. This pattern confidence expresses the precision ingredient $\left(P_{\text {positive }} /\left(P_{\text {positive }}+P_{\text {negative }}\right)\right)$ and the recall ingredient $\left(\left[\log _{2}\left(P_{\text {new }}+1\right)\right]^{\alpha}\right)$ of the pattern $P$. and $\alpha$ is the percentage of correct pairs in new ones. When $P_{\text {new }}$ and $\alpha$ are larger, the pattern confidence is higher too. If $P_{\text {new }}$ or $\alpha$ is equal to 0 , the pattern confidence goes back to its initial definition in [7].

\section{Experiments}

In this section we give the experiment results of SPG and compare them with the results of DIPRE. We use the sub-collections ranging from \#29 to \#39 of the CWT100g (Chinese Web Test collection with 100GB web pages) corpus for training and use the sub-collections ranging from \#60 to \#63 for testing. This paper uses the following pairs as initial seeds in terms of "is part of" conceptual relationship.

Table 1. Initial Chinese Seed List

\begin{tabular}{cc}
\hline 吉林 (Ji Lin) & 长春 (Chang Chun) \\
中国 (China) & 黑龙江 (Hei Longjiang) \\
北京 (Bei Jing) & 海淀 (Hai Dian) \\
陕西 (Shan Xi) & 西安 (Xi An) \\
上海 (Shang Hai) & 静安 (Jing An) \\
\hline
\end{tabular}

The results of experiments by running three iterations can be seen in Table 2 .

Table 2. Pairs and Patterns in every iteration

\begin{tabular}{c|c|c|c|c}
\hline & \multicolumn{2}{|c|}{ SPG } & \multicolumn{2}{c}{ DIPRE } \\
\hline \multirow{2}{*}{ Iterations } & Tuples & Patterns & Tuples & Patterns \\
1 & 5 & 28 & 5 & 21 \\
2 & 57 & 51 & 31 & 33 \\
3 & 171 & 169 & 20 & 25 \\
\hline
\end{tabular}

In the first run, both the two systems use the same five seeds as initial input and the last two runs use the seeds extracted from the previous iteration. Column 3 and 4 show the number of valid patterns. Seen from the experiment results, SPG can extract more conceptual pairs than DIPRE does from the same sub-collections.

Then we need to consider the ability of SPG in constructing the conceptual relationship between concepts. We use all the patterns obtained into the test collection, respectively. Because the test collection is fixed, the system extracting MORE correct pairs has the higher recall. Their precisions are easily evaluated manually. 
SPG has the better coverage than DIPRE because SPG can get more conceptual pairs. On the other hand, the precisions also shows that SPG does better than DIPRE: the precision of SPG in three iterations keeps about $90 \%$ with slightly fall, while the DIPRE's precision drops very quickly, and arrives at 50\% in its third iteration. After the experiment on testing corpus, SPG system totally obtain 1,504 pairs at last, and 1,358 of them are correct (precision $=90.29 \%$ ); DIPRE obtains 588 pairs, and 384 of them are correct (precision $=60.20 \%$ ). The SPG patterns have higher performance.

\section{Conclusion}

In this paper, we propose a system named SPG for determining whether two concepts satisfy the "is part of" relation in given context. We introduce a bi-sequence alignment algorithm in bio-informatics to capture clearer and more understandable patterns. And we also define a new confidence evaluating method for patterns. After training the SPG system, the experiment results show that our system performs better than DIPRE in terms of coverage and precision.

As mentioned above, our study aims at serving the retrieval model based on concepts. Therefore the pattern set ought to give the support to determine the relationship between two concepts in contexts. The pattern set should be useful in many web pages and the precision should be guaranteed. For a long-term goal, recognizing the accurate relationships definitely bring IR huge benefits, but to the performance of current system, it is still a hard job. The key points of our future work lies in developing an advanced SPG system and extending the method proposed in this paper to the recognition of other relationships.

Acknowledgments. This work is supported by NSFC Major Research Program 60496326: Basic Theory and Core Techniques of Non Canonical Knowledge.

\section{References}

1. Agichtein, E., and Gravano, S.: Snowball: Extracting relations from large plain-text collections. In Proc. of the 5th ACM International Conference on Digital Libraries (2000)

2. Brin, S.: Extracting patterns and relations from the World Wide Web. In Proc. of the 1998 International Workshop on the Web and Databases (1998)

3. Etzioni O., Cafarella M., Downey D. and Popescu A.M. et al: Methods for DomainIndependent Information Extraction from the Web: An Experimental Comparison. In Proc. of the AAAI Conference (2004)

4. Genest, D. and Chenin, M. A Content-Search Information Retrieval Process Based on Conceptual Graphs. Knowledge and Information Systems Journal. (2005) Vol. 8, 292-309

5. Lin W., Yangarber R., Grishman R.: Bootstrapped Learning of Semantic Classes from Positive and Negative Examples. In Proc. of the ICML-2003 Workshop on the Continuum from Labeled to Unlabeled Data, Washington DC (2003)

6. Michael S., B. Morgenstern, and J. Stoye. Divide-and-conquer multiple alignment with segment-based constraints. Bioinformatics. (2003) 19(2): 189-195

7. Thelen M. and Riloff E.: A Bootstrapping Method for Learning Semantic Lexicon using Extraction Pattern Contexts. In Proc. of the 2002 Conference on Empirical Methods in Natural Language Processing (2002) 